\title{
Semiquantitative Method
}

National Cancer Institute

\section{Source}

National Cancer Institute. Semiquantitative Method. NCI Thesaurus. Code C94980.

As colloquially used, a designation for a simpler quantitative method that contrasts with a more rigorous one used for a similar purpose. Often the terminology, semiquantitative analysis, is used in imaging as a synonym for usage of the standardized uptake value. 